\title{
ESTRATEGIA DIDÁCTICA PARA LA PRODUCCIÓN DE TEXTOS ESCRITOS EN ESPAÑOL COMO LENGUA EXTRANJERA
}

\author{
Didactic Strategy for the Production of Written Texts in Spanish as a Foreign \\ Language \\ Virgen Arelys Ferrer Miyares, Dra. C. \\ Universidad de Oriente, Cuba \\ https://orcid.org/0000-0002-2444-7697 \\ virgenfm@uo.edu.cu
}

Palabras claves: Español Lengua Extranjera, Producción Escrita, Aprendizaje Cooperativo.

Keywords: Spanish as a Foreign Language, Written Text, Cooperative Learning, Reflection.
Recibido: 22 de junio de 2018

Aceptado: 09 de julio de 2018

\section{RESUMEN}

La habilidad de escribir, en la enseñanza de español como segunda lengua es la que presenta progresos más lentos en el aprendizaje de los estudiantes extranjeros y, las limitaciones radican, en insuficiencias que presenta su enseñanza. Esta habilidad resulta muy compleja para su tratamiento en el aula al exigir de una preparación por parte del docente que posibilite su desarrollo, consciente de los obstáculos que debe vencer el estudiante para lograr el éxito que presupone su dominio que se evidencia cuando puede comunicar de forma escrita diferentes tipos de textos a partir de situaciones diversas. En este trabajo se presenta una estrategia didáctica dirigida al perfeccionamiento del proceso enseñanza aprendizaje del español como lengua extranjera, con énfasis en la producción de textos escritos. Por lo que se tuvo presente la activación de este proceso, a través de la producción cooperativa de textos escritos en español como lengua extranjera encaminada a desarrollar en los estudiantes la producción y la eficacia donde la metacognición de sus procederes y conocimientos es fundamental.

\begin{abstract}
In the teaching of Spanish as second language, the ability of writing is the one that presents the slowest progress in the learning of foreign students, and the limitations are in the insufficiencies that its teaching presents. This skill is very complex for its treatment in the classroom. It demands preparation of the teacher for its development, with the awareness of the obstacles that the student must overcome to be successful which presuppose its mastery that are shown when they can communicate themselves in written way different types of texts from many situations. In this project work a didactic strategy is presented, addressed to the improvements of the teaching learning process of Spanish as foreign language, with emphasis in the production of written texts. That is why; the activation of the process was taken into account, through the cooperative production of written texts in Spanish as foreign language. The purpose is to develop in students the production and efficiency where the self-cognition of its procedures and knowledges is fundamental.
\end{abstract}




\section{INTRODUCCIÓN}

Escribir en una lengua que no es la materna, es un complejo proceso donde el estudiante planifica, escribe y revisa en las diferentes etapas y al considerarlo de esta manera, dialécticamente se enriquece en esa recursividad, en ese ir y venir, que no es ni puede ser lineal.

Incluso algunos autores comparten la idea de que la escritura por sus complejidades, se puede comparar con la resolución de problemas.

Los estudiantes que vienen a Cuba a estudiar una carrera universitaria ya conocen un mundo y realidades que no son iguales a las de Cuba, pero que en sus inicios es necesario tener presente para conectar con lo ya conocido por ellos, y, en la medida que avanzan en su comprensión de la nueva realidad, ellos van incorporando lo que aprenden, van comparando con lo que ha sido su mundo y van sintetizando esos conocimientos en sus textos escritos.

Se enfrentan a una nueva realidad: una lengua que necesitan aprender para tener éxito en sus estudios académicos, pero algo fundamental es que no deben darse contradicciones que pongan en cuestionamiento su cultura de origen porque el objetivo de su tránsito por esta realidad debe lograrse que se produzca como proceso de intercambio de saberes, culturas, valores, sentimientos, actitudes, integración, lo cual le permitirá desempeñar su profesión en su país o en otro lugar con una visión amplia que le permita asumir realidades diferentes, con respeto.

Cuando el estudiante llega al aula preparatoria de español, debe adquirir casi al unísono las cuatro habilidades comunicativas. Este proceso sucede con bastante rapidez, ya que cuenta con muy poco tiempo entre su preparación en la lengua meta y su acceso al currículo universitario de la carrera seleccionada.

La ventaja que posee es el dominio maduro de su lengua (o lenguas) de origen, lo cual le facilita el reto de aprender una nueva realidad. En este trabajo se presenta una estrategia didáctica dirigida al perfeccionamiento del proceso enseñanza aprendizaje del español como lengua extranjera, con énfasis en la producción de textos escrito.

\section{DESARROLLO}

\section{Algunos referentes para el desarrollo del proceso de producción de textos escritos en la enseñanza del español como lengua extranjera.}

La Didáctica de la Escritura se ocupa de la enseñanza de la construcción textual y su proceso se ha interesado, junto a otras ciencias que estudian el lenguaje, por el tratamiento metodológico en el devenir histórico.

En la enseñanza de las lenguas extranjeras y del español como lengua extranjera la escritura no siempre ha sido objeto de interés. En la actualidad esta didáctica recibe influencias de la psicología cognitiva, de las técnicas de creatividad o los métodos de solución de problemas y la heurística.

La enseñanza de español lengua extranjera (ELE) se realiza en los diferentes países con un estudiantado de procedencia diversa, lo que lleva a cuestionarse también el nivel de familiarización de este con la cultura escrita y lleva a distinguir (Cassany, 2009:50-51) las características de los estudiantes que proceden de culturas predominantemente orales y los que proceden de culturas altamente alfabetizadas, donde la lectura y la escritura constituye una actividad de realización habitual y continua.

La metodología propuesta por el enfoque cognitivo comunicativo y sociocultural brinda las vías para poder proceder en la enseñanza del discurso, así como profundiza en el tratamiento didáctico de los tres componentes que lo integran: el discurso, la cognición y la sociedad y se orienta al desarrollo de la competencia, cognitiva, comunicativa y sociocultural, que Angelina Roméu (2003:13) define como "una configuración psicológica que comprende las capacidades cognitivas y metacognitivas para comprender y producir significados, los conocimientos acerca de las estructuras lingüísticas y discursivas y las capacidades para interactuar en diversos contextos socioculturales, con diferentes fines y propósitos.

Teniendo como presupuesto el enfoque histórico - cultural, es posible precisar que lo central en el proceso de enseñanza, en este caso de la producción textual, consiste en asegurar las condiciones para que el estudiante crezca, mediante la actividad conjunta, a un nivel superior; partiendo de lo que aún no puede hacer solo, que llegue a lograr un dominio independiente de sus funciones. Significa colocar al estudiante como centro de atención a partir del cual se debe proyectar el proceso pedagógico.

Que, para el estudiante, implica utilizar los elementos de los que dispone en su personalidad, a saber: su historia académica, sus intereses cognoscitivos, sus motivos estudiar, su emocionalidad, sus saberes, su cultura, entre otros, 
en relación con los que aporta el grupo de clase, involucrando a los propios estudiantes en la construcción de las condiciones más favorables para el aprendizaje.

Del profesor Cassany, D. (2009) se toma su definición de que: "La composición escrita es una finalidad comunicativa y una técnica instrumental para desarrollar la adquisición del español como lengua extranjera." (p. 63.). E insiste en la necesidad de que sea visto el acto de escribir como lo que es una compleja tarea en la cual el estudiante debe advertir sus procesos para aprender.

Propone que para producir un texto eficaz son necesarios los conocimientos, las destrezas y los valores; además la necesidad de que el estudiante concientice que la lengua oral y la escrita son fenómenos de comunicación con sus particularidades que obligan a que en esta última sea imprescindible la elaboración del borrador. (p.52.).

El método comunicativo que se ha empleado en el proceso de enseñanza aprendizaje del español como lengua extranjera tiene particularidades favorables, pero no ha logrado llevar el desarrollo del texto escrito a los niveles deseados.

La investigadora Roméu, A. (2003). en su enfoque cognitivo, comunicativo y sociocultural aborda los diferentes componentes del mismo, y por supuesto la construcción de textos, que es necesario especificarlo más para el caso de las lenguas extranjeras.

Por lo que se concibe el proceso de producción de textos escritos en estudiantes no hispanohablantes como un interproceso de naturaleza didáctica que se define como un sistema de continuados momentos interconectados y dirigidos a la exploración, invención y elaboración de significados comprensibles desde las referencias semánticas, constructivas y sociales de la lengua de origen, contextualizadas a la lengua que se aprende, que permiten la comunicación escrita, en condiciones de interacción y aprendizaje cooperativo propiciadores de la productividad. Ferrer M, V., (2015).

Esta estrategia se basa en el trabajo cooperativo planteado en el desarrollo del proceso de producción de textos escritos en español como lengua extranjera significa la organización que realiza el docente en grupos de estudiantes formados de diferentes maneras que responden, en condiciones de ayuda mutua colectiva, a la resolución de problemas que plantean las diferentes tareas comunicativas escritas. Ferrer M, V. (2015).

\section{Caracterización de la estrategia didáctica.}

Se asume el criterio de estrategia didáctica de Márquez R, A. (2000) al definirla como sistema dinámico y flexible que se pone en práctica de forma gradual, permitiendo una evaluación sistemática y la intervención activa de sus participantes y en la cual son significativos, no solo los resultados, sino también el desarrollo del proceso.

Esta definición adaptable a la estrategia didáctica que se presenta es reveladora de un sistema de acciones proyectadas para lograr la transformación y permite enriquecer mediante la reflexión los conocimientos.

Posibilita ajustar la toma de decisiones, recobrar el conocimiento sobre la enseñanza y trazar acciones intencionadas dirigidas a repercutir en los componentes didácticos:

Objetivo, contenido, método, medios y en la evaluación, con el propósito de perfeccionar el proceso de enseñanza aprendizaje del objeto en cuestión que se ha investigado, en una situación dada:

- Para la implementación de esta estrategia didáctica son importantes los siguientes requerimientos en el orden científico metodológico:

- Aseguramiento de las condiciones previas que implican la preparación académica de los docentes en los conocimientos lingüísticos y didácticos para interiorizar e implementar procederes novedosos en la enseñanza de ELE.

Nivel de motivación de los docentes frente al proceso de perfeccionamiento del proceso de enseñanza aprendizaje de ELE direccionado al proceso de producción de textos escritos.

\section{Concepción general de la estrategia didáctica.}

En la concepción de la estrategia se tuvo en cuenta el intercambio con 11 especialistas que se dedican a este tipo de enseñanza, la han impartido en diferentes cursos, tanto en Cuba como en el extranjero o han estudiado en algún momento esta materia, con el propósito de atender sus criterios y consideraciones para perfeccionar la estrategia. 
Objetivo general de la estrategia: Ofrecer procederes didácticos a los profesores para la enseñanza aprendizaje del español como lengua extranjera, que garanticen el desarrollo del proceso de producción de textos escritos de los estudiantes no hispanohablantes.

\section{Etapa de diagnóstico y sensibilización.}

Objetivo: Diagnosticar las necesidades de los estudiantes y sensibilizar a los docentes para perfeccionar el proceso de producción de textos escritos en ELE.

Los requerimientos relacionados con el docente, responden a:

- Nivel de preparación y disposición, para interiorizar y potenciar el proceso de producción de textos escritos en la enseñanza de ELE.

- Dominio de las características de los estudiantes no hispanohablantes.

- Dominio de los aspectos esenciales de la didáctica de ELE, la concepción del trabajo metodológico para la enseñanza del proceso de producción de textos escritos con el propósito de potenciar la preparación de los docentes y la socialización en los colectivos y espacios posibilitadores de divulgar las experiencias obtenidas.

En el caso de los estudiantes debe tenerse presente:

- La disposición para solucionar la tarea propuesta en trabajo cooperativo en grupos.

- El conocimiento de la importancia de la interacción grupal necesaria en el proceso de producción de textos escritos y de la variedad textual.

Se aplicaron diferentes técnicas con características participativas y que propician la reflexión e instrumentos que posibilitaron un intercambio positivo para el debate científico y metodológico.

Los talleres metodológicos realizados fueron:

- Primer Taller. Para que el estudiante produzca un texto escrito en ELE procedo así... (Debate abierto).

- Segundo Taller. ¿Corregir errores en las producciones escritas de mis estudiantes? (Debate y reflexión)

Los talleres permitieron desarrollar la cultura del intercambio, el respeto por los criterios del otro u otra y a través de la explicación, de la argumentación de las ideas y prácticas desarrolladas valorar las posibilidades del colectivo en situaciones de intercambio e interacción grupal, porque esto es fundamental para lograr desarrollarlo en los estudiantes; al igual que contribuir a la reflexión constante y sistemática de la práctica pedagógica como elemento imprescindible de la mejora sistemática del proceso de enseñanza aprendizaje.

Las reflexiones se realizaron a través de preguntas relacionadas con las interrogantes que puede hacerse el estudiante que pretende producir un texto escrito y las que se hace el docente para que este proceso esté orientado, organizado y controlado por él y por los propios estudiantes de manera efectiva:

\begin{tabular}{|c|c|}
\hline Docente & Estudiante \\
\hline ¿Por qué desean y necesitan escribir? & ¿Necesito escribir? ¿Por qué? \\
\hline ¿Qué y a quién escribir? & ¿Qué y a quién necesito escribirle? \\
\hline ¿Qué requieren saber para escribir? & ¿Qué necesito saber? \\
\hline ¿Qué relación tienen con el destinatario? & ¿Qué relación tengo con el destinatario? \\
\hline $\begin{array}{l}\text { ¿Cuál es la intención y la finalidad de lo } \\
\text { que escribirán? }\end{array}$ & $\begin{array}{l}\text { ¿Cuál es mi intención y qué quiero obtener de la } \\
\text { persona a la que le escribo? }\end{array}$ \\
\hline $\begin{array}{l}\text { ¿Qué tipo de texto deben escribir para } \\
\text { lograr el fin? }\end{array}$ & $\begin{array}{l}\text { ¿Qué tipo de texto debo escribir para lograr el } \\
\text { fin? }\end{array}$ \\
\hline $\begin{array}{l}\text { ¿Cuál es el estilo y la forma elocutiva } \\
\text { conveniente para esa finalidad? }\end{array}$ & $\begin{array}{l}\text { ¿De qué manera expreso lo que quiero escribir y } \\
\text { qué palabras utilizo para lograr lo que quiero? }\end{array}$ \\
\hline $\begin{array}{l}\text { ¿Cómo procedo para que el proceso de } \\
\text { producción se se desarrolle } \\
\text { adecuadamente? }\end{array}$ & $\begin{array}{l}\text { ¿Cómo desarrollo mis ideas, de qué, quién o } \\
\text { quiénes necesito ayuda o intercambiar lo que } \\
\text { tengo proyectado para que lo que llegue a la } \\
\text { persona (o personas) a quien (o a quienes) dirijo } \\
\text { lo escrito sea entendible y cumpla mi propósito? }\end{array}$ \\
\hline $\begin{array}{l}\text { ¿Le ofrezco la posibilidad de interactuar } \\
\text { con otros mientras escribe? }\end{array}$ & $\begin{array}{l}\text { Si tuviera la posibilidad de interactuar con otros } \\
\text { (u otras personas), mientras escribo, la tarea a } \\
\text { realizar sería más amena, menos tensa e } \\
\text { interesante. }\end{array}$ \\
\hline
\end{tabular}


¿Qué indicadores le brindo para guiarse al escribir y revisar el texto que necesitan?

¿Cómo reconocerán los errores?
¿Escucho lo que me aconsejan, intercambio con los miembros del grupo y tengo la posibilidad de consultar fuentes diversas para enriquecer mi producción escrita y la de mis compañeros y compañeras de grupo?

¿Qué guía poseo para escribir y revisar el texto? ¿Se ajusta el texto al tipo de discurso seleccionado?

¿El texto es coherente?

¿Es original el texto escrito?

¿Cómo han sido usadas las estructuras gramaticales (verbos, preposiciones, conjunciones, etc.)?

¿Cumple mi texto con las normas ortográficas españolas que conozco?

¿He logrado un texto fluido?

¿Cuántas veces será necesario autorrevisar el texto producido?

¿Qué me van a evaluar y cómo participaré en esa evaluación?

¿Cómo contribuyo al mejoramiento de las producciones escritas de mi grupo?

¿Cómo mejorar mis textos escritos?

¿Incorporaré para mi nueva revisión todo lo que se revisó en el aula o lo que me resulta más complejo o no logrado en mi texto producido anteriormente?

¿Participar en mi propia evaluación y en la de otros me resulta útil?

Los razonamientos de los docentes los llevan a plantear que las actividades están adecuadamente diseñadas, porque tienen presente la necesidad de los estudiantes de interactuar activamente en grupos y parejas, durante el proceso de producción escrita y participar en la evaluación de sus logros y dificultades, lo cual les permite crecer. Mostraron criterios favorables hacia el dinamismo que le brinda esta manera de proceder en la actividad en el aula que por lo general se desarrolla de manera silenciosa y crea un ambiente misterioso y tenso que no dispone a los estudiantes de forma favorable a una tarea compleja como la de producir textos escritos en ELE.

En la estrategia se da la posibilidad de ofrecerle tratamiento a textos que responden a tipologías diferentes, a partir de las situaciones, las intenciones y la finalidad que se persigue al escribir, de lo que el estudiante debe tomar conciencia, es decir que el tipo de texto dependerá de su intención y la finalidad; por tanto, el lenguaje también responderá a ello.

\section{Etapa de ejecución}

Objetivo: Ejecutar las acciones diseñadas en la estrategia para el proceso en cuestión.

Para conocer el desempeño del docente en el desarrollo de las actividades de producción de textos escritos en ELE y el comportamiento de los estudiantes con esta manera de hacer del docente, se proponen las siguientes acciones dirigidas al:

- Diseño e implementación de actividades de producción textual escrita interactiva en la que el estudiante en interacción con sus compañeros y con el texto busque y descubra los recursos lingüísticos y constructivos que le sirvan para la elaboración de su propio texto.

- Diseño y elaboración, bajo la guía del profesor, de textos escritos formales necesarios en su vida profesional y cotidiana (llenados de planillas, solicitud de empleo, currículo, anuncios, promociones laborales, solicitud de contratos como empleadores, convocatorias de empleo, informe de investigación sobre temas de la profesión o de preocupaciones colectivas), donde se aprecie el carácter de conciliación sociopragmática que se propone.

- Organización del aula en grupos para desarrollar la producción cooperativa de textos escritos en ELE.

- Respeto del profesor a la cultura de origen del estudiante, a través del empleo del procedimiento de la simulación y promover el respeto y apropiación sin conflictos antagónicos de la cultura de la lengua meta que lleven al rechazo y a la no aceptación de los valores de cada una. 
- Clima favorable en el aula propiciador del trabajo cooperativo que genera la reflexión creativa y plantea compromiso con el grupo y con su propio aprendizaje.

- Desarrollo de acciones en ambientes no aúlicos: teatros, parques, museos, hoteles, restaurantes, oficinas consulares y de inmigración, mercados, hospitales, terminales de ómnibus, aeropuertos, visita a escuelas, a hogares cubanos, departamentos de profesores, centro de diagnóstico y orientación, gabinetes de orientación, entre otros.

- Presentación y orientación de materiales contentivos de actividades que responden a diferentes tipologías textuales, incluyendo la visual, la audiovisual y textos periodísticos.

- Tratamiento al léxico a partir de la variante cubana, contextualizada.

- Atención a las interferencias propias del que aprende una lengua extranjera.

- Producción reflexivo - creativa que se desarrolla cuando el estudiante interactúa directamente con el texto que está elaborando, a partir de cuestionamientos diferentes que responden al contenido, la adecuación al lector, su organización y cierre.

- Desarrollo del proceso de textualización.

- Socialización en el grupo de los resultados de la marcha del proceso de producción para reflexionar en cuanto a la construcción y reconstrucción del proceso.

\section{Etapa de evaluación}

Objetivo: Evaluar el resultado obtenido durante el proceso de producción de textos escritos por estudiantes no hispanohablantes de español como lengua extranjera.

Acciones:

- Evaluación concebida como proceso, presente durante toda la producción del texto escrito en ELE.

- Que el estudiante lleve un registro de sus logros, aciertos y desaciertos.

- Tratamiento didáctico del error como fuente de aprendizaje de una lengua extranjera.

- Aplicación de la heteroevaluación, coevaluación y autoevaluación.

- El docente lleva un registro que recoge los logros, dificultades e interferencias que presenta el proceso. Desde esta perspectiva se contribuye al desarrollo de valores como la responsabilidad, la honestidad.

- Entrega de una guía con los indicadores para que el estudiante pueda coevaluarse y autoevaluarse.

- Diseño de un boletín para una revista creada por los propios estudiantes cuyo consejo editorial valora los mejores trabajos para su publicación en el centro.

Valoración de la aplicación de la estrategia didáctica para la producción de textos escritos en español como lengua extranjera.

- Las tareas que conducen a la producción de textos escritos promovieron el desarrollo de otras habilidades, pues siempre se parte de una situación comunicativa que requiere de actividad oral de ellos, a partir de preguntas, la observación de fotos, la visualización de un vídeo o la audición que sirven para desarrollar el interés por el tema, familiarizarse y sistematizar también el léxico relacionado con la temática y brindar información y orientación sobre algún aspecto necesario para resolver la tarea. La constancia, la motivación, los intereses por aprender, el intercambio propiciador de un ambiente constructivo posibilitó que crecieran y se apropiaran de los conocimientos y habilidades para elaborar un texto escrito.

- Realizaron una tarea integradora que les posibilitó hacer la selección de un tema de interés, sobre el cual debían documentarse en diferentes fuentes que incluyeron el análisis bibliográfico, la entrevista, visitas a museos, a lugares de interés cultural, educacional e histórico, revisión de materiales, entre otras; para elaborar un informe que se ajustara a la estructura de este tipo de documento y exponerlo de forma oral ante el colectivo estudiantil y docente; lo que formaba parte del desarrollo de sus habilidades al tener que preguntar y responder preguntas a quien las formulara y usar, a su vez, la tecnología para la exposición.

- El resultado de la tarea integradora demostró que el intercambio con el grupo, con los docentes del colectivo de ELE y otros profesores de la carrera, que fungieron como tutores de esos proyectos, fue favorable porque posibilitó el desarrollo de las diferentes habilidades comunicativas.

- Los estudiantes reconocen que el trabajo en equipo o con otra persona es fructífero porque: en equipo se intercambian experiencias, conocimientos, ideas, opiniones, hay un aprendizaje grupal, dinámico., se garantiza un mejor perfeccionamiento entre los miembros del equipo, más cooperatividad, responsabilidad, se intercambia y se llega a una idea mejor y se facilita el aprendizaje y la comprensión de la materia, se aprende mucho trabajando en dúo, el grupo influye mucho en el proceso de enseñanza aprendizaje, contribuye a un mejor desarrollo y responsabilidad, el aprendizaje se da de diferentes maneras y permite la participación 
colectiva, se desarrollan habilidades cognoscitivas. El equipo contribuye al aprendizaje, al intercambio de conocimientos, brinda orientación y las ayudas necesarias, se juntan las ideas y se llega a una conclusión.

Las sugerencias planteadas permitieron elaborar dos programas para el currículo optativo/electivo que han sido impartidos de la siguiente manera:

- Didáctica del Español como lengua extranjera. Impartido en el 5 año de la Licenciatura en Educación Español Literatura, en tres cursos desde 2015-2017, con resultados positivos, porque los estudiantes expresaron que el curso les brinda herramientas didácticas para enfrentarse a la enseñanza de personas no hispanohablantes, el respeto por las culturas de otros países y de otras lenguas, los métodos en la enseñanza de ELE, vías para enfrentar un colectivo de estudiantes que hablan otras lenguas y quieren o necesitan aprender el español, concientizaron las complejidades de este proceso de enseñanza aprendizaje de ELE, les dieron respuestas a interrogantes que tenían y contribuye a su preparación para instruir y educar dentro y fuera del país a estudiantes no hispanohablantes.

- Escribir en Español. Impartido en la carrera Pedagogía Psicología a estudiantes angolanos en el curso 20142015, con muy buenos resultados porque posibilitaron el trabajo cooperativo en equipo, haciéndose énfasis en la interdependencia positiva entre sus miembros, la interacción cara a cara, la responsabilidad de cada miembro y la evaluación grupal.

\section{Principales transformaciones logradas en los estudiantes:}

- La producción de textos escritos en español como lengua extranjera con mayor calidad, a partir del desarrollo del trabajo cooperativo.

- El logro de metas comunes, en las cuales la colaboración y la interacción permitieron el desarrollo de las habilidades comunicativas, en condiciones favorables.

- Demostraron con sus producciones textuales, su desarrollo cultural y psicopedagógico para afrontar favorablemente la solución de los problemas profesionales: estudios de caso, estrategias de intervención, trabajos científicos estudiantiles; y con ello contribuir al desarrollo de la personalidad del profesional de educación.

- Crecimiento personal al asumir el trabajo cooperativo solidario como aspecto necesario para la vida.

\section{Principales transformaciones logradas en los docentes:}

- Permitió perfeccionar la orientación del proceso de producción textual del español como lengua extranjera, a partir del desarrollo del aprendizaje cooperativo.

- La interacción grupal permite el desarrollo de todas las habilidades comunicativas en los estudiantes y contribuye a que el estudiante se enfrente al proceso con menos temores.

\section{CONCLUSIÓN}

La estrategia didáctica sustentada en la interacción grupal posibilitó que los estudiantes no hispanohablantes elaboraran textos escritos en español como lengua extranjera adecuadamente.

La producción cooperativa de textos escritos en español como lengua extranjera propicia a desarrollar en los estudiantes la producción y la eficacia donde la metacognición de sus procederes y conocimientos es fundamental.

\section{BIBLIOGRAFÍA}

1. Cassany, D. (2009). La composición escrita en E/LE. En Monográficos marco ELE. Expolingua 1999, núm. 9, pp. 47-66.

2. Ferrer Miyares, V. (2016). Variante metodológica interactivo-reflexiva para la producción de textos escritos en español como lengua extranjera. Evento Provincial 10mo. Congreso Internacional de Educación Superior. Universidad 2016.

3. Roméu Escobar, A. (2003). "Enseñanza de la comprensión y producción de textos científicos como problema interdisciplinario". En Folleto Curso de Superación. Ciudad de La Habana, Pedagogía 2003.

4. Vigotsky, L. S. (1998). Pensamiento y lenguaje. Ciudad de La Habana: Editorial Pueblo y Educación. 